\title{
Ultrasound Assessment of Human Meniscus
}

${ }^{1,2}$ Viren T, ${ }^{1,4}$ Honkanen JTJ, ${ }^{1,4}$ Danso EK, ${ }^{1,3}$ Rieppo L, ${ }^{1}$ Korhonen RK, ${ }^{1,4}$ Töyräs J

1. Department of Applied Physics, University of Eastern Finland, Kuopio, Finland

2. Cancer Center, Kuopio University Hospital, Kuopio, Finland

3. Research Unit of Medical Imaging, Physics and Technology, Faculty of Medicine, University of Oulu, Oulu, Finland

4. Diagnostic Imaging Center, Kuopio University Hospital, Kuopio Finland

10 The final authenticated version is available online at:

https://doi.org/10.1016/i.ultrasmedbio.2017.01.020

Submitted to Ultrasound in Medicine and Biology, in September 2016

\section{Corresponding author:}

Tuomas Viren, Ph.D.

Department of Applied Physics

University of Eastern Finland

Yliopistonranta 1

70211 Kuopio, Finland 


\section{ABSTRACT}

The aim of the present study was to evaluate the applicability of ultrasound imaging for quantitative assessment of human meniscus in vitro. Meniscus samples $(n=26)$ were harvested from 13 knee

5 joints of non-arthritic human cadavers. Subsequently, three locations (anterior, center and posterior) from each meniscus were imaged with two ultrasound transducers (frequencies 9 and 40 $\mathrm{MHz}$ ) and quantitative ultrasound parameters were determined. Furthermore, partial least squares (PLS) regression analysis was applied for ultrasound signal to determine the relations between ultrasound scattering and the meniscus integrity. Significant correlations between measured and predicted meniscus composition and mechanical properties were obtained $\left(R^{2}=0.38-0.69, p<0.05\right)$. The relation of conventional ultrasound parameters with integrity of meniscus was weaker. To conclude, ultrasound imaging showed potential for evaluation of meniscus integrity. Higher ultrasound frequency combined with multivariate analysis of ultrasound backscattering was found to be the most sensitive for evaluation of the meniscus integrity.

15 Key Terms: Quantitative ultrasound imaging; Osteoarthritis; Composition; Meniscus 


\section{INTRODUCTION}

Menisci are two fibrocartilaginous crescent-shape discs located between femoral condyles and proximal tibia in the medial and lateral sides of the knee joint (Fox et al. 2012; Messner and Gao 1998). Menisci are mainly composed of water (60-70\%) and collagens (15-25\%, mainly type I) (Fox

5 et al. 2012). Proteoglycans, noncollagenous proteins, glycoproteins and cells account for the rest of the meniscus weight (Fox et al. 2012). Meniscus has a highly organized structure which gives the tissue its characteristic mechanical properties such as high tensile stiffness and strength, low compression stiffness and low permeability (Fox et al. 2012; Masouros et al. 2008). The main functions of the meniscus are to stabilize the knee joint, distribute loads between the femur and tibia and participate on the lubrication of the joint (McDermott et al. 2008). The changes in the structure and composition of the meniscus (caused by e.g. joint injury) may lead to deterioration of the mechanical function of the meniscus. This may subsequently lead to extensive loading of the articular cartilage and instabilization of the whole knee joint (McDermott and Amis 2006). The changes in the knee joint function can initiate the degeneration of the articulating surfaces, which eventually leads to development of osteoarthritis (Englund and Lohmander 2004). Currently, meniscal injuries can be treated with surgical techniques, such as sutures and darts or in the case of more severe injuries meniscus allograft transplantation and partial or complete meniscectomy (Roos et al. 1998; Sihvonen et al. 2013; Van Der Straeten et al. 2016). Unfortunately, the effectiveness of the current surgical techniques, especially meniscectomy, in the treatment of meniscus injuries is questionable (Roos et al. 1998; Sihvonen et al. 2013; Van Der Straeten et al. 2016). Quantitative tools capable of accurate determination of the extent and severity of meniscus injuries would be important when developing novel surgical techniques aiming to restore the meniscal function. 
Currently, injuries and degeneration of menisci are diagnosed using clinical examination, magnetic resonance imaging (MRI), computed tomography (CT) arthrography and arthroscopy (Fox 2007; Huysse et al. 2008). However, current clinical MRI or CT techniques may not be optimal for the quantitative assessment of the integrity of menisci. The relatively low resolution,

5 high costs and limited availability of clinical MRI, and radiation exposure and the need of contrast agents to improve the soft tissue contrast of clinical CT imaging hinder the potential of these techniques for the accurate evaluation of meniscal pathologies. Furthermore, neither MRI nor CT imaging can be conducted during arthroscopic surgery. Thus, the final evaluation of the status of the meniscal injuries during the repair operation is based on the arthroscopic evaluation. Arthroscopic assessment of the integrity of the joint consists of visual inspection and mechanical palpation of the articulating surfaces (Brittberg and Winalski 2003). However, this method is highly subjective and the diagnosis is dependent on the experience of the operating surgeon (Spahn et al. 2009). Thus, novel quantitative arthroscopic imaging techniques are needed.

High frequency external ultrasound imaging has been reported to be a sensitive method for evaluation of meniscal injuries and function (Akatsu et al. 2015; Rowland et al. 2016). In previous studies, ultrasound imaging has been used to evaluate tears, cysts and mechanical function of the meniscus (Friedman et al. 2001; Nogueira-Barbosa et al. 2015; Rowland et al. 2016; Wareluk and Szopinski 2012). As the ultrasound imaging is a safe and widely available technique, screening of the meniscal damages after the joint injuries might be possible. However, only limited areas of the meniscus are visible with external ultrasound, which decreases the diagnostic value of ultrasound investigation (Akatsu et al. 2015). Furthermore, ultrasound attenuation and scattering in the soft tissues surrounding the knee joint significantly complicate the interpretation and quantification of the ultrasound images (Friedman et al. 2001). Recently, ultrasound arthroscopy was introduced for high resolution ultrasound imaging of the articular cartilage and subchondral 
bone during an arthroscopic surgery (Kaleva et al. 2011; Liukkonen et al. 2013). Ultrasound arthroscopy technique has been reported to be sensitive for the changes in the structure and composition of articular cartilage related to development of osteoarthritis (Liukkonen et al. 2014; Virén et al. 2009). In previous studies, ultrasound arthroscopy has been used to evaluate cartilage 5 injuries in knee and shoulder joints in vivo (Kaleva et al. 2011; Liukkonen et al. 2014; Puhakka et al. 2016). However, the capability of ultrasound imaging to quantify the compositional and structural changes related to meniscus degeneration is unknown. Furthermore, due to highly organized layered structure of the meniscus, the quantitative ultrasound parameters related to bulk properties of the tissue may not reflect accurately the integrity of the complex tissue, and thus, more sophisticated techniques for analyzing depth dependent ultrasound scattering are needed. In previous studies, multivariate partial least squares (PLS) regression analysis has been applied for analysis of near infrared spectroscopy and optical coherence tomography data measured from articular cartilage and meniscus (Afara et al. 2015; Ala-Myllymäki et al. 2016; Puhakka et al. 2015). PLS regression analysis is commonly used to detect relations between noisy predictor variables and one or more response variables. PLS analysis has also been used to correlate the light backscattering with the composition and mechanical properties of cartilage and menisci (Ala-Myllymäki et al. 2016; Puhakka et al. 2015). However, the applicability of PLS regression analysis of ultrasound backscattering for the determination of structural and compositional parameters from the meniscus is unknown.

The aim of the present study was to evaluate the potential of ultrasound imaging for the assessment of meniscus degeneration and to introduce a multivariate technique for analysis of ultrasound backscattering in meniscus. 


\section{MATERIALS AND METHODS}

\section{Sample preparation}

Lateral and medial meniscus samples $(n=26)$ were harvested from left knee joints of human cadavers ( $n=13$, age 24-76 years, 12 male, 1 female) with no history of joint disease (National

5 Agency of Medicolegal Affairs, Helsinki, Finland; permission 1781/32/200/01). Subsequently, menisci were wrapped in gauze soaked in physiological phosphate buffered saline (PBS; Euroclone, Paignton-Devon, UK) and stored at $-25{ }^{\circ} \mathrm{C}$ until the experiment. Before the ultrasound measurements the menisci were thawed in water bath at room temperature and immersed in PBS containing inhibitors of proteolytic enzymes ( $5 \mathrm{mM}$ disodium EDTA and $5 \mathrm{mM}$ benzamidine $\mathrm{HCl}$ ).

\section{Ultrasound measurements}

Clinical ultrasound device (ClearView Ultra, Boston Scientific Corporation, San Jose, CA, USA) with two high frequency $(9 \mathrm{MHz}$ and $40 \mathrm{MHz}$; Ultra Ice and Atlantis pro, Boston Scientific, San Jose, USA, respectively) intravascular ultrasound (IVUS) imaging catheters was used in the present study. The technical details of the imaging system have been described in our previous papers (Liukkonen et al. 2013; Virén et al. 2009). During the measurements the samples were kept in degassed PBS, which contained inhibitors of proteolytic enzymes ( $5 \mathrm{mM}$ disodium EDTA and $5 \mathrm{mM}$ benzamidine $\mathrm{HCl}$ ). Then the ultrasound catheter was manually positioned over the meniscus and the angle between ultrasound transducer and meniscus surface was adjusted to obtain maximum reflection. Subsequently, radiofrequency (RF) ultrasound signal from ten consecutive ultrasound acquisitions was recorded and digitized (sampling frequency $250 \mathrm{MHz}$ ) with custom-made software (LabView version 8.2, National Instruments Corporation, Austin TX, USA) and a digital oscilloscope (LeCroy Corporation, Wave Runner 6051A, Chestnut Ridge, NY, USA). For each meniscus the ultrasound measurements were conducted in three anatomical locations: anterior, central and posterior 
section of the meniscus. The measurement locations were marked with ink to match the locations of ultrasound measurements and the reference analyses. Minimum of four successful measurements were conducted in each measurement location. For each repeated measurement (consisting of ten ultrasound images) one ultrasound acquisition with the highest reflection from

5 meniscus surface, i.e. the most optimal angle between ultrasound transducer and meniscus surface, was selected for further analysis. Finally, three measurements with the highest reflection from meniscus surface were selected for the final analysis. After the ultrasound measurements the samples were stored at $-25^{\circ} \mathrm{C}$ until reference measurements.

To calculate the absolute values of ultrasound reflection and backscattering parameters a reference reflection was measured from a polished steel plate (for $40 \mathrm{MHz}$ ultrasound transducer) and a rubber phantom (for $9 \mathrm{MHz}$ ultrasound transducer) with known reflection coefficients of $93 \%$ and $10 \%$, respectively. Due to the high output of $9 \mathrm{MHz}$ ultrasound transducer, the reflection measured from steel reference plate exceeded the dynamic range of the used oscilloscope and thus reference reflector with lower reflection coefficient was used for $9 \mathrm{MHz}$ ultrasound transducer. The reference reflection was measured using various distances $(2.5-10 \mathrm{~mm}$, 56 measurement distances and 1.3-7.2 mm, 52 measurement distances, for $9 \mathrm{MHz}$ and $40 \mathrm{MHz}$, respectively) between the ultrasound catheter and the saline-reference interface. The reflected ultrasound pulse was windowed using rectangular window (width $=0.64 \mu$ s and $0.4 \mu \mathrm{s}$ for $9 \mathrm{MHz}$ and $40 \mathrm{MHz}$ ultrasound, respectively). For each ultrasound measurement the reference reflection, recorded at the nearest distance to that of the reflection from the saline-meniscus interface, was selected for the analysis. 


\section{Quantitative ultrasound parameters}

Reflection coefficient $(R)$, integrated reflection coefficient $(I R C)$, apparent integrated backscattering coefficient $(A I B)$ and ultrasound roughness index $(U R I)$ were determined with both ultrasound transducers for each measurement location as described in earlier studies (figure 1) (Chérin et al.

5 1998; Saarakkala et al. 2004; Virén et al. 2009). Briefly, for calculation of the reflection parameters $(R$ and $I R C)$ one scan line perpendicular to the meniscus surface was selected from each recorded ultrasound image. This was done by selecting the scanline with the highest reflection from salinemeniscus interface. $R$ was calculated from the ultrasound signal reflected from the meniscus surface in the time domain, whereas IRC was determined in the frequency domain (Virén et al. 2009; Virén et al. 2010). The final value was obtained as an average of three repeated measurements. AlB was determined in the frequency domain at the same scan line as the reflection parameters (Virén et al. 2009). URI was calculated from the meniscus surface profiles each consisting of 21 points (10 points from each side of the point closest to the IVUS probe) (Virén et al. 2009). The trend arising from the measurement geometry and the natural curvature of the meniscus surface was removed before determination of URI by fitting a $4^{\text {th }}$ order polynomial in to the measured profile and subtracting the fit from the original measurements.

Before the calculation of quantitative parameters RF ultrasound signal was filtered using digital band pass filters $\left(9 \mathrm{MHz}\right.$ transducer: $6^{\text {th }}$ order, pass band $1.5-20 \mathrm{MHz}, 40 \mathrm{MHz}$ transducer: $15^{\text {th }}$ order, pass band $15-80 \mathrm{MHz}$ ). Before the calculation of $R$ and IRC the reflected ultrasound pulse was windowed using a Hamming window (width $=0.64 \mu$ s and $0.4 \mu$ s for $9 \mathrm{MHz}$ and $40 \mathrm{MHz}$ ultrasound, respectively) and before the calculation of $A / B 1.85 \mu$ s and $1.94 \mu \mathrm{s}$ rectangular windows were selected under the meniscus surface for $9 \mathrm{MHz}$ and $40 \mathrm{MHz}$ ultrasound transducers, respectively. Furthermore, to investigate the depth dependence of ultrasound 
scattering three consecutive $A I B$ widows $(A I B s: 0.64 \mu \mathrm{s}$ and $0.4 \mu \mathrm{s} ; A I B m$ : $0.64 \mu \mathrm{s}$ and $0.4 \mu \mathrm{s}$; and AlBd: $0.74 \mu \mathrm{s}$ and $1.23 \mu \mathrm{s}$, for $9 \mathrm{MHz}$ and $40 \mathrm{MHz}$, respectively) were selected (figure 1).

\section{Biomechanical measurements}

Biomechanical data used in the present study was extracted from our previous study with the same samples and measurement sites (Danso et al. 2015). The details of biomechanical measurements are described in that study. Briefly, a custom-made mechanical testing device with cylindrical flat ended steel indenter $($ diam. $=1.19 \mathrm{~mm})$, high resolution $(0.1 \mu \mathrm{m})$ actuator $(\mathrm{PM1A1798}$; Newport Corporation, Irvine, CA), load cell (Model 31/AL311AR, Honeywell, Columbus, OH, USA, resolution: $0.005 \mathrm{~N}$ ) and measurement and data acquisition software (LabView; national Instruments, Austin, TX) was used for biomechanical testing. Four compressive steps were applied using a ramp rate of $100 \% / \mathrm{s}$ and a relaxation criterion of $<10 \mathrm{~Pa} / \mathrm{min}$. Subsequently, the equilibrium modulus $\left(E_{e q}\right)$ (linear fit to stress-strain ratios in equilibrium points from steps 2 to 4 ) and instantaneous modulus ( $E_{\text {inst }}$ ) (linear part of stress-strain curve in step 4) were calculated by assuming Poisson's ratio of 0.1 and 0.5 , respectively.

\section{Biochemical analysis}

The biochemical data used in the present study was extracted from a previous study with the same samples (Danso et al. 2015). For the analysis $1 \mathrm{~mm}$ thick section was cut from a site adjacent to the ultrasound and biomechanical measurement site. Water content $\left(\mathrm{H}_{2} \mathrm{O}\right)$ of the meniscus samples was determined using the freeze drying method. Samples were first immersed in the PBS for 4 hours and weighed. The measurement was repeated three times and the results were averaged. Subsequently, lyophilized for 72 hours and then weighted to determine the dry weight of the samples. Water content was determined as ratio of dry and wet weight subtracted from $100 \%$. Subsequently, 
hydroxyproline (HyPro, related to collagen concentration) and uronic acid (UA, related to proteoglycan concentration) contents of the samples were determined. To determine the HyPro and UA contents the sections prepared from each measurement location were incubated for 3 hours in $60^{\circ} \mathrm{C}$ with a mixture of $1 \mathrm{mg} / \mathrm{ml}$ concentration of papain in $150 \mathrm{mM}$ of sodium acetate including

$550 \mathrm{mM}$ Cys-HCL and $5 \mathrm{mM}$ EDTA. Next, the papain-digested sections were freeze dried and hydrolysed and the HyPro content was determined spectrophotometrically (Schwartz et al. 1985). Finally, the UA content was quantified from the ethanol-precipitated residue of the digested sections (Blumenkratz and Asboe-Hansen 1973). For each sample, the biochemical analysis were repeated three times and the results were averaged. Variation in the section size was compensated by normalizing the results with the wet weight of the section.

\section{Digital Densitometry}

To determine the histological integrity of the samples Safranin-O stained sections (thickness $=3 \mu \mathrm{m}$ ) were prepared at the site of ultrasound and biomechanical measurements. Subsequently, the distribution of proteoglycans was quantified by imaging optical density $(O D)$ of the Safranin-O stained sections with a digital densitometry system (DD) consisting of light microscope (Axiolmager M2, Carl Zeiss, Oberkochen, Germany) with monochromatic light source and a 12-bit CCD camera ( $\mathrm{CH} 250$, Photometrics, Tucson, AZ, USA). The system was calibrated using neutral density filter (Schott, Mainz, Germany). Finally, $O D$ was determined for each site as an average of three sections.

\section{PLS regression multivariate analysis}

Multivariate PLS regression analysis was applied to predict the composition and mechanical properties of the meniscus based on measured ultrasound backscattering. PLS regression analysis is used to detect relations between predictor variable and one or more response variables. $E_{\text {eq, }}, E_{\text {inst }}$, 
$\mathrm{H}_{2} \mathrm{O}$ content, $\mathrm{OD}, U A$ content and HyPro content were used as response variables in the analysis. In the present study, two different sets of predictor variables were used. To determine the predictor variables, $1.85 \mu$ s and $1.94 \mu$ s rectangular windows were selected under the meniscus surface for 9 $\mathrm{MHz}$ and $40 \mathrm{MHz}$ ultrasound transducers, respectively. First, Hilbert envelope was calculated for the 5 ultrasound signal and z-scores calculated from depth dependent ultrasound backscattering intensity were used as predictor variables in the PLS regression models $\left(P L S_{E}\right.$, figure 1$)$. To determine the second set of predictor variables, power spectrum was calculated for the windowed ultrasound signal. Subsequently, power spectrum of measured signal was normalized with the power spectrum of a reference reflection from a polished steel plate. Finally, z-scores determined from $-12 \mathrm{~dB}$ bandwidth (7.4-10.8 MHz and 32.0-44.7 $\mathrm{MHz}$ for $9 \mathrm{MHz}$ and $40 \mathrm{MHz}$ ultrasound, respectively) of ultrasound backscattering spectrum were selected for the PLS regression analysis (PLSs).

Ten-fold cross-validation technique was used to build and validate the PLS prediction models (Lorber and Kowalski 1990). In ten-fold cross-validation, the samples are randomly divided into ten groups. In turn, each group of samples are left out from the analysis and their properties are predicted based on PLS regression models built using the rest of the samples. Further, competitive adaptive reweighted sampling was used to select the most relevant predictor variables for the PLS regression models. The algorithm evaluates the importance of individual predictor variables based on their regression coefficients. The variables with small regression coefficients are considered less important and removed from the models in an iterative process (Li et al. 2009). The maximum number of PLS components was limited to six to avoid overfitting. The criteria for the optimal model were to maximize the coefficient of determination $\left(R^{2}\right)$ and minimize the root mean square error of cross-validation (RMSECV) between the measured and predicted response variables. Custom made Matlab (2014a, MathWorks Inc, Natick, MA USA) script and libPLS toolbox (Li et al. 2014) was used to preprocess the ultrasound data and to create the PLS models. 


\section{Statistical analysis}

Statistical analyses were conducted with SPSS software (SPSS 19, IBM, Chicago, IL, USA). The relationships between quantitative ultrasound parameters and mechanical properties or composition of the meniscus were determined with Spearman's rank correlation coefficient. The

5 statistical significance between the ultrasound parameter values measured from different anatomical locations was tested with Freeman analysis of variance. In the pair-wise analysis, adjusted $p$-values were used to account the bias caused by multiple testing. Linear dependencies between quantitative ultrasound parameters and menisci properties determined with reference techniques was tested with mixed-model analysis. The mixed-model accounts the correlations and clustering within the dataset. In the mixed model analysis, the joint in which the measurements were conducted was set to random variable and meniscus properties, location and side were set to fixed variables. The reproducibility of the ultrasound measurements was determined as a coefficient of variation (CV) (Glüer et al. 1995) and as a standardized coefficient of variation (sCV) (Fournier et al. 1997). To determine $s C V$ the $C V$ values should be standardized using the ratio of range and mean of parameter values, where the range is determined by multiplying the population standard deviation by factor of 4 (Fournier et al. 1997; Njeh et al. 2000). However, as the population mean and standard deviation were not available for quantitative ultrasound parameters the values determined in the present study were used in the determination of $s C V$. To estimate the clinical usability of the technique Least Significant Change values (LSC) were calculated for each ultrasound parameter (Glüer 1999). In the calculation of $L S C$ the $s C V$ was used as an approximation for the precision error of the technique. The level of statistical significance was set to $p<0.05$.

\section{RESULTS}


The mean and standard deviations of the quantitative ultrasound parameters determined from different locations (anterior, center and posterior) of medial and lateral menisci are presented in the figure 2. For $9 \mathrm{MHz}$ ultrasound transducer, significant $(p<0.05)$ site dependent variation was observed in the ultrasound reflection parameters ( $R$ and $I R C)$. However, ultrasound backscattering $5(A I B, A I B s, A I B m$ and $A I B d)$ and surface roughness $(U R I)$ values did not show statistically significant variation between the locations (figure 2). Backscattering of $40 \mathrm{MHz}$ ultrasound varied significantly $(p<0.05)$ between the measurement locations whereas the ultrasound reflection and roughness parameters did not (figure 2). Average $R, I R C, U R I$ and $A I B$ determined with $9 \mathrm{MHz}$ ultrasound for meniscus were $6.3 \pm 2.2 \%,-26.5 \pm 3.9 \mathrm{~dB}, 25.7 \pm 9.9 \mu \mathrm{m}$ and $-35.9 \pm 5.7 \mathrm{~dB}$. Average ultrasound parameters values determined with $40 \mathrm{MHz}$ ultrasound for meniscus were $7.5 \pm 2.2 \%,-28.6 \pm 2.8 \mathrm{~dB}$, $25.2 \pm 9.6 \mu \mathrm{m}$ and $-39.3 \pm 3.0 \mathrm{~dB}$ for $R, I R C, U R I$ and $A I B$, respectively.

Moderate but significant correlations were found between the quantitative ultrasound parameters and the composition and mechanical properties of menisci (table 1). With 9 $\mathrm{MHz}$ ultrasound, statistically significant correlation was detected between $R, I R C, A I B m$ or $A l B d$ and HyPro and between $R$ or IRC and UA content of the menisci (table 1). With $9 \mathrm{MHz}$ ultrasound, no significant correlations were detected between $A I B, A I B s$ or $U R I$ and, composition and mechanical properties of menisci (table 1). With $40 \mathrm{MHz}$ ultrasound, modest but significant correlations were found between $I R C$ and HyPro and $R$ or $I R C$ and $O D$ of the menisci. Furthermore, moderate but significant correlation was found between URI and HyPro (table 1). Finally, moderate but significant correlations were detected between the ultrasound backscattering parameters $(A|B, A| B s, A l B m$ and $A / B d)$ and, composition and mechanical properties of meniscus (table 1). The reproducibility of the ultrasound parameters (CV and $S C V$ ) varied between 0.1-27.3\% (table 2). LSC values determined for the ultrasound parameters varied between $0.2-51.1 \%$ (table 2). 
Mixed model was used to determine the linear relations between quantitative ultrasound parameters and composition and mechanical properties of the meniscus. For $9 \mathrm{MHz}$ ultrasound, significant linear relation was found between the collagen content (HyPro) of meniscus and ultrasound reflection (IRC, $p=0.03$ ). Furthermore, significant linear relations were found

5 between ultrasound reflection and the meniscus $\mathrm{H}_{2} \mathrm{O}$ and $U A$ contents $(p \leq 0.01)$ and between the $A I B$ or $U R I$ and HyPro $(p \leq 0.02$ ) (table 3 ). For $40 \mathrm{MHz}$ ultrasound, surface roughness parameter URI was found to be significantly related to mechanical properties of the meniscus $\left(E_{e q}\right.$ and $\left.E_{\text {inst }}\right)$ and HyPro content of the meniscus $(p<0.01)$. Furthermore, linear relation was found between the mean $O D$ of Safranin-O stained sections and $A I B(p=0.05)$ (table 3$)$. ultrasound backscattering signal ( $\mathrm{PLS}$ E and $\mathrm{PLS} S$ ) correlated with meniscus composition and mechanical properties determined with reference techniques (table 4). Significant correlations were detected between predictions based on PLS regression analysis of $40 \mathrm{MHz}$ ultrasound backscattering envelope $\left(R^{2}=0.38-0.69, p<0.05\right)$ or power spectrum $\left(R^{2}=0.30-0.51, p<0.05\right)$ and mechanical properties and composition of meniscus (table 4). For $9 \mathrm{MHz}$ ultrasound, only modest correlations $\left(R^{2}<0.3\right)$ were detected between predictions determined form ultrasound backscattering envelope or power spectrum and meniscus composition and mechanical properties (table 3).

\section{DISCUSSION}

The feasibility of quantitative ultrasound imaging for the evaluation of the composition and mechanical properties of the meniscus was investigated. Quantitative ultrasound parameters describing the integrity of the meniscus were calculated and correlated with meniscus composition and mechanical properties determined with reference techniques. Furthermore, multivariate PLS 
analysis of ultrasound backscattering was introduced for the determination of composition and mechanical properties of the meniscus.

Utilization of multivariate PLS regression model for the analysis of ultrasound backscattering improved significantly the evaluation of the integrity of human meniscus. Statistically

5 significant correlations were detected between the measured composition and mechanical properties of meniscus and the predicted values obtained with multivariate PLS regression analysis of ultrasound backscattering signal. Especially, $E_{\text {inst }}, E_{e q}, O D, \cup A$ and HyPro were associated with the backscattering of $40 \mathrm{MHz}$ ultrasound. The results are in line with an earlier study in which PLS analysis of the depth dependent OCT signal was found to significantly improve the quantification of the osteoarthritis related changes in the highly organized layered articular cartilage when compared with bulk optical backscattering or attenuation (Puhakka et al. 2015). In the present study, no significant differences were detected between PLS predictions calculated based on depth dependent ultrasound backscattering or backscattering spectrum. However, higher ultrasound frequency ( $40 \mathrm{MHz}$ ) was found to be more suited for PLS regression analyses than $9 \mathrm{MHz}$ ultrasound.

15 This might be due to the higher axial resolution and lower noise level of $40 \mathrm{MHz}$ ultrasound transducer as compared to $9 \mathrm{MHz}$ ultrasound.

Despite the significant $R^{2}$ values, the RMSECV values remained high in the PLS analysis. This may diminish the clinical applicability of the technique for patient specific assessment of meniscus properties. However, as the number of samples available in the present study was limited, 20 the created multivariate PLS-model may not yet be optimal, and increasing the number of samples used to create the PLS model would likely improve the accuracy of the predictions. In addition, application of similar techniques for the pre-processing of measured ultrasound signal, than used to enhance the sensitivity of the PLS analysis of light backscatter, might improve the sensitivity of 
the analysis (Puhakka et al. 2015; Rieppo et al. 2012). Finally, application different variable selection methods might also improve the PLS model and, thus, decrease the RMSECV values. However, as the PLS analysis of the ultrasound backscattering signal was found to improve the correlations between ultrasound scattering and composition and mechanical properties of meniscus, the 5 technique warrants further investigation.

Due to the dependency between the measurement locations, mixed model analysis was used to determine the linear relations between quantitative ultrasound parameters and composition and mechanical properties of the meniscus. In previous studies, collagen was reported to be the main scatterer of ultrasound in articular cartilage (Töyräs et al. 1999). Thus, the significant linear relation between ultrasound reflection or backscattering and HyPro content detected in present study was expected. Furthermore, surface roughness indicating the integrity of the superficial collagen network of the meniscus was related to the meniscus mechanical properties and HyPro content. In addition, the UA content $\mathrm{H}_{2} \mathrm{O}$ content and $O D$ of Safranin-O stained sections (i.e. proteoglycan concentration) were found to be related to ultrasound reflection and ultrasound backscattering parameters. In previous studies, the correlation between ultrasound parameters and proteoglycan content measured from articular cartilage was reported to be insignificant (Pellaumail et al. 2002; Saarakkala et al. 2004). However, as the proteoglycan content as well as water content has been reported to change due to the degeneration of the articular cartilage or meniscus the relations detected in the present study may be due to general degeneration of meniscus structure rather than changes in the proteoglycan or water content (Buckwalter et al. 2005; Herwig et al. 1984).

In the present study, significant correlation was detected between quantitative ultrasound parameters and composition and mechanical properties of meniscus. Several previous 
studies report similar correlations between quantitative ultrasound parameters and composition or mechanical properties of articular cartilage (Liukkonen et al. 2013; Saarakkala et al. 2006; Viren et al. 2011). However, the quantitative ultrasound evaluation of the meniscus has been sparse. The previous studies on ultrasound imaging have been mostly focused on the qualitative evaluation of

5 the integrity of the meniscus or quantitative measurement of the excursion of the meniscus under loading (Akatsu et al. 2015; Friedman et al. 2001; Rowland et al. 2016). However, these studies have been conducted using conventional external ultrasound imaging systems and no relations of measured ultrasound backscattering to mechanical properties or composition of meniscus have been reported. To our knowledge, there is only one previous study in which quantitative ultrasound parameters have been correlated to mechanical properties of the meniscus (Yasura et al. 2007). In that study, intact and enzymatically degraded porcine menisci was investigated with quantitative ultrasound system and moderate correlation was detected between the ultrasound reflection from meniscus surface and apparent Young's modulus of the meniscus. Furthermore, the intensity of ultrasound reflection was reported to decrease after the enzymatic degradation of the meniscus collagens but remain unchanged after digestion of proteoglycans. In the present study, we found a significant correlation between the ultrasound reflection ( $R$ or $I R C$ with $9 \mathrm{MHz}$ ultrasound) and UA or HyPro contents of the meniscus (i.e. proteoglycan and collagen content, respectively).

Compared to measurements conducted with the $40 \mathrm{MHz}$ transducer, slightly better reproducibility ( $C V$ and $s C V$ ) of ultrasound reflection parameters was achieved when $9 \mathrm{MHz}$ ultrasound transducer was used. This indicates that the $9 \mathrm{MHz}$ ultrasound transducer might not be as prone to positioning errors as the $40 \mathrm{MHz}$ ultrasound transducer. Compared to ultrasound reflection and backscattering parameters the reproducibility of URI was relatively low with both ultrasound transducers. Due to the rotating measurement geometry, the length of evaluated meniscus surface profile was limited (21 measurement points corresponding roughly to $1 \mathrm{~mm}$ ) 
which may have decreased the reproducibility of URI. Furthermore, the lateral resolution of the ultrasound system and, thus the determined $U R I$ value, is dependent on the distance between the imaging catheter and meniscus surface which further decreases the reproducibility of the determination of URI. The present findings are in line with previous studies (Virén et al. 2009; Virén et al. 2010). $L S C$-values determined for $R, I R C$ and $A I B$ were significantly lower than those determined for URI. LSC parameter can be used to estimate the smallest change in the quantitative parameter value that is considered statistically significant. Furthermore, if the difference in the parameter values determined for normal and pathological tissues is known LSC can be used to estimate the techniques ability to discriminate between normal and pathological tissues. Earlier, only one study reporting a quantitative ultrasound evaluation of meniscus degeneration has been published. In that study the change in the ultrasound reflection on the meniscus surface degreased more than $60 \%$ after the enzymatic digestion of meniscus superficial collagen network (Yasura et al. 2007). Despite the enzymatic digestion of the meniscus collagen might not perfectly mimic osteoarthritis related degeneration of the tissue the results indicate that the changes in the ultrasound reflection related to meniscus pathologies might be greater than the LSC-values determined for ultrasound reflection in the present study. However, further clinical studies are needed to confirm the statistical power of the ultrasound evaluation of the meniscus.

Ultrasound reflection from saline-meniscus interface was in the same range as the values previously determined with similar ultrasound system for human knee articular cartilage (6.7 $\pm 2.5 \%$ and $-23.8 \pm 4.1 \mathrm{~dB}$ for $R$ and $I R C$, respectively) (Liukkonen et al. 2013). This was expected as the constituents of meniscus are similar to those of articular cartilage. However, the backscattering parameters determined for meniscus were higher than those previously reported for human articular cartilage $(A I B=-45.5 \pm 4 \mathrm{~dB}$ ) (Liukkonen et al. 2013). Even though there are similarities in the composition of the meniscus and articular cartilage, the structure of these tissues are different. 
In the meniscus, the collagen network is predominantly oriented parallel to the meniscus surface, whereas in articular cartilage the orientation of the collagen network is parallel to cartilage surface, random, and perpendicular to cartilage surface in the superficial, the middle and the deep layers of cartilage, respectively. As the orientation of the collagen network has been shown to significantly 5 affect the ultrasound backscattering (Inkinen et al. 2015), the difference in echogenicity between cartilage and meniscus might be due to differences in collagen network structure.

Two different ultrasound catheters with different center frequencies were used in the present study. Higher frequency ultrasound enables higher image resolution which might improve the detection of small changes related to meniscus pathologies. However, due to rapid increase in ultrasound attenuation as a function of frequency, the penetration of high frequency ultrasound into biological tissues is limited. Thus, the optimal ultrasound frequency is a tradeoff between image resolution and desired penetration depth. In the present study, ultrasound reflection parameters $(R$ and $I R C$ ) determined with $9 \mathrm{MHz}$ ultrasound transducer were more sensitive for the changes in meniscus composition as compared to those determined using $40 \mathrm{MHz}$ ultrasound. Due to the larger beam width and longer pulse length, the $9 \mathrm{MHz}$ ultrasound might be less sensitive than the high frequency $40 \mathrm{MHz}$ ultrasound transducer for the inaccuracies in the positioning of the transducer and small roughness at the meniscus surface. To simulate the clinical conditions, all the ultrasound measurements were done by holding the ultrasound catheter manually above the meniscus surface which may have led to suboptimal positioning of the ultrasound catheter. The suboptimal positioning of the ultrasound catheter (i.e. non-perpendicular incidence of ultrasound at meniscus surface) likely generated uncertainty especially in the measurements of ultrasound reflection coefficient. This might explain the lack of correlation between the ultrasound reflection and meniscus composition in measurements done with $40 \mathrm{MHz}$ ultrasound transducer. This assumption is also supported by the lower reproducibility of $40 \mathrm{MHz}$ ultrasound reflection parameters as 
compared to measurements done with $9 \mathrm{MHz}$ ultrasound. URI and backscattering measured with the $40 \mathrm{MHz}$ ultrasound frequency were found to be more sensitive for changes in the composition and mechanical properties of the menisci than those determined using $9 \mathrm{MHz}$ ultrasound. Due to short pulse length, extensive backscattering and high lateral resolution of $40 \mathrm{MHz}$ ultrasound, the 5 surface roughness and backscattering characteristics of the meniscus could be determined more accurately with $40 \mathrm{MHz}$ transducer than with $9 \mathrm{MHz}$ ultrasound. This is in line with an earlier study where high ultrasound frequency and small spot size of ultrasound beam were found perquisites for sensitive evaluation of the degenerative changes in articular cartilage (Kaleva et al. 2009).

Conducting reproducible and sensitive ultrasound measurements during a clinical arthroscopic surgery is challenging. Thus, to enable fast and straightforward assessment of the meniscus and articulating surfaces the ultrasound imaging techniques should be robust. Furthermore, for the clinical applications, the visualization of the underlying subchondral bone and measurement of cartilage thickness would be valuable. Thus, clinical applications might favor lower ultrasound frequency at the expense of lower resolution. However, based on the results of the present study, lower ultrasound frequencies might not be sensitive enough for the determination of material and mechanical properties of meniscus based on the ultrasound backscattering measurement. Thus, for the clinical application the highest possible ultrasound frequency that still can be used to visualize the subchondral bone should be selected.

To conclude, quantitative ultrasound imaging was shown to be potential technique for evaluation of the integrity of the human meniscus in vitro. Furthermore, PLS regression analysis of ultrasound backscattering was shown to be superior to quantitative ultrasound parameters in detecting variation in meniscus composition and mechanical properties. However, further 
optimization of the preprocessing of ultrasound signal might further improve the accuracy of PLS prediction model in the evaluation of the composition and mechanical properties of the meniscus.

\section{ACKNOWLEDGMENTS}

Financial support from the Sigrid Juselius Foundation, strategic funding of the University of Eastern

5 Finland, National Doctoral Programme of Musculoskeletal Disorders and Biomaterials (TBDP), Kuopio University Hospital (VTR projects 1V276, 1V090 and 5654156) and Academy of Finland (projects 267551 and 269315) are acknowledged.

\section{REFERENCES}

Afara IO, Hauta-Kasari M, Jurvelin JS, Oloyede A, Töyräs J. Optical absorption spectra of human articular cartilage correlate with biomechanical properties, histological score and biochemical composition. Physiol Meas 2015;36:1913-1928.

Akatsu Y, Yamaguchi S, Mukoyama S, Morikawa T, Yamaguchi T, Tsuchiya K, Iwasaki J, Akagi R, Muramatsu Y, Katsuragi J, Fukawa T, Endo J, Takahashi K, Sasho T. Accuracy of high-resolution ultrasound in the detection of meniscal tears and determination of the visible area of menisci. J Bone Joint Surg Am 2015;97:799-806.

Ala-Myllymäki J, Honkanen JT, Töyräs J, Afara IO. Optical spectroscopic determination of human meniscus composition. J Orthop Res 2016;34:270-8.

Blumenkratz N, Asboe-Hansen G. New method for quantitative determination of uronic acids. Anal Biochem 1973;54:484-489.

Brittberg M, Winalski C. Evaluation of cartilage injuries and cartilage repair. J Bone Jt Surg 2003;85:58-69. 
Buckwalter JA, Mankin HJ, Grodzinsky AJ. Articular cartilage and osteoarthritis. Instr Course Lect $2005 ; 54: 465-480$.

Chérin E, Saed A, Laugier P, Netter P, Berger G. Evaluation of acoustical parameter sensitivity to agerelated and osteoarthritic changes in articular cartilage using 50-MHZ ultrasound. Ultrasound Med Biol 1998;24:341-354.

Danso EK, Mäkelä JTA, Tanska P, Mononen ME, Honkanen JTJ, Jurvelin JS, Töyräs J, Julkunen P, Korhonen RK. Characterization of site-specific biomechanical properties of human meniscusImportance of collagen and fluid on mechanical nonlinearities. J Biomech Elsevier Ltd, 2015;48:1499-1507.

Englund M, Lohmander LS. Risk factors for symptomatic knee osteoarthritis fifteen to twenty-two years after meniscectomy. Arthritis Rheum 2004;50:2811-2819.

Fournier B, Chappard C, R C, Berger G, Laugier P. International Original Article Quantitative Ultrasound Imaging at the Calcaneus Using an Automatic Region of Interest. Osteoporos Int 1997;363-369.

15 Fox AJS, Bedi A, Rodeo SA. The basic science of human knee menisci: structure, composition, and function. Sports Health 2012;4:340-51.

Fox MG. MR Imaging of the Meniscus: Review, Current Trends, and Clinical Implications. Magn Reson Imaging Clin N Am 2007;15:103-123.

Friedman L, Finaly K, Jurriaans E. Ultrasound of the knee. Skelet Radiol 2001;30:361-377.

Glüer C. Monitoring Skeletal Changes by Radiological Techniques. J Bone Miner Res 1999;14:19521962.

Glüer C, Blake G, Lu Y, Blunt B, Jergas M, Genant H. Accurate assessment of precision errors: how to 
measure the reproducibility of bone densitometry techniques. Osteoprosis Int 1995;5:262270.

Herwig J, Egner E, Buddecke E. Chemical changes of human knee joint menisci in various stages of degeneration. Ann Rheum Dis 1984;43:635-40.

Huysse WCJ, Vestraere KL, Verdonk PC, R V. Meniscus Imaging. Semin Musculoskelet Radiol 2008;12:318-333.

Inkinen SI, Liukkonen J, Tiitu V, Virén T, Jurvelin JS, Töyräs J. Ultrasound backscattering is anisotropic in bovine articular cartilage. Ultrasound Med Biol 2015;41:1958-1966.

Kaleva E, Töyräs J, Jurvelin JS, Viren T, Saarakkala S. Effects of ultrasound frequency, temporal sampling frequency, and spatial sampling step on the quantitative ultrasound parameters of articular cartilage. IEEE Trans Ultrason Ferroelectr Freq Control 2009;56:1383-1393.

Kaleva E, Virén T, Saarakkala S, Sahlman J, Sirola J, Puhakka J, Paatela T, Kröger H, Kiviranta I, Jurvelin JS, Töyräs J. Arthroscopic Ultrasound Assessment of Articular Cartilage in the Human Knee Joint: A Potential Diagnostic Method. Cartilage 2011;2:246-53.

Li H-D, Xu Q-S, Liang Y-Z. libPLS: An Integrated Library for Partial Least Squares Regression and Discriminant Analysis. Peer J Prepr 2014;2:e190v1:3-6.

Li H, Liang $\mathrm{Y}, \mathrm{Xu} \mathrm{Q}$, Cao D. Key wavelengths screening using competitive adaptive reweighted sampling method for multivariate calibration. Anal Chim Acta 2009;648:77-84.

Liukkonen J, Hirvasniemi J, Joukainen A, Penttilä P, Virén T, Saarakkala S, Kröger H, Jurvelin JS, Töyräs J. Arthroscopic ultrasound technique for simultaneous quantitative assessment of articular cartilage and subchondral bone: an in vitro and in vivo feasibility study. Ultrasound Med Biol 2013;39:1460-8. 
Liukkonen J, Lehenkari P, Hirvasniemi J, Joukainen A, Virén T, Saarakkala S, Nieminen MT, Jurvelin JS, Töyräs J. Ultrasound Arthroscopy of Human Knee Cartilage and Subchondral Bone in Vivo. Ultrasound Med Biol 2014;40:2039-2047.

Lorber A, Kowalski BR. Alternatives to cross validation estimation of the number of factors in multivariate calibration. Appl Spectrosc 1990;44:1464-1470.

Masouros SD, McDermott ID, Amis AA, Bull AMJ. Biomechanics of the meniscus-meniscal ligament construct of the knee. Knee Surgery, Sport Traumatol Arthrosc 2008;16:1121-1132.

McDermott ID, Amis AA. The consequences of meniscectomy. J Bone Joint Surg Br 2006;88:154956.

McDermott ID, Masouros SD, Amis AA. Biomechanics of the menisci of the knee. Curr Orthop 2008;22:193-201.

Messner K, Gao J. The menisci of the knee joint. Anatomical and functional characteristics, and a rationale for clinical treatment. J Anat 1998;193:161-178.

Njeh CF, Hans D, Li J, Fan B, Fuerst T, He YQ, Tsuda-Futami E, Lu Y, Wu CY, Genant HK. Comparison of six calcaneal quantitative ultrasound devices: Precision and hip fracture discrimination. Osteoporos Int 2000;11:1051-1062.

Nogueira-Barbosa MH, Gregio-Junior E, Lorenzato MM, Guermazi A, Roemer FW, Chagas-Neto FA, Crema MD. Ultrasound assessment of medial meniscal extrusion: a validation study using MRI as reference standard. AJR Am J Roentgenol 2015;204:584-588.

Pellaumail B, Watrin A, Loeuille D, Netter P, Berger G, Laugier P, Saed A. Effect of articular cartilage proteoglycan depletion on high frequency ultrasound backscatter. Osteoarthr Cartil 2002;10:535-541. 
Puhakka J, Afara IO, Paatela T, Sormaala MJ, Timonen MA, Viren T, Jurvelin JS, Töyräs J, Kiviranta I. In Vivo Evaluation of the Potential of High-Frequency Ultrasound for Arthroscopic Examination of the Shoulder Joint. Cartilage 2016;7:248-255.

Puhakka PH, te Moller NCR, Afara IO, Mäkelä JTA, Tiitu V, Korhonen RK, Brommer H, Virén T, Jurvelin JS, Töyräs J. Estimation of articular cartilage properties using multivariate analysis of optical coherence tomography signal. Osteoarthr Cartil 2015;23:2206-2213.

Rieppo L, Saarakkala S, Närhi T, Helminen HJ, Jurvelin JS, Rieppo J. Application of second derivative spectroscopy for increasing molecular speci fi city of fourier transform infrared spectroscopic imaging of articular cartilage. Osteoarthr Cartil Elsevier Ltd, 2012;20:451-459.

Roos H, Lauren M, Adalberth T, Roos EM, Jonsson K, Lohmander LS. Knee osteoarthritis after meniscectomy: Prevalence of radiographic changes after twenty-one years, compared with matched controls. Arthritis Rheum 1998;41:687-693.

Rowland G, Mar D, Mclff T, Nelson J. Evaluation of meniscal extrusion with posterior root disruption and repair using ultrasound. Knee Elsevier B.V., 2016;23:627-630.

15 Saarakkala S, Laasanen MS, Jurvelin JS, Töyräs J. Quantitative ultrasound imaging detects degenerative changes in articular cartilage surface and subchondral bone. Phys Med Biol 2006;51:5333-46.

Saarakkala S, Töyräs J, Hirvonen J, Laasanen MS, Lappalainen R, Jurvelin JS. Ultrasonic quantitation of superficial degradation of articular cartilage. Ultrasound Med Biol 2004;30:783-792.

Schwartz DE, Choi Y, Sandell LJ, Hanson WR. Quantitative analysis of collagen, protein and DNA in fixed, paraffin-embedded and sectioned tissue. Histochem J 1985;17:655-663.

Sihvonen R, Paavola M, Malmivaara A, Itälä A, Joukainen A, Nurmi H, Kalske J, Järvinen TLN. Arthroscopic partial meniscectomy versus sham surgery for a degenerative meniscal tear. $\mathrm{N}$ 
Engl J Med 2013;369:2515-24.

Spahn G, Klinger HM, Hofmann GO. How valid is the arthroscopic diagnosis of cartilage lesions? Results of an opinion survey among highly experienced arthroscopic surgeons. Arch Orthop Trauma Surg 2009;129:1117-1121.

Töyräs J, Rieppo J, Nieminen MT, Helminen HJ, Jurvelin JS. Characterization of enzymatically induced degradation of articular cartilage using high frequency ultrasound. Phys Med Biol 1999 [cited 2016 Jul 7];44:2723-2733.

Van Der Straeten C, Byttebier P, Eeckhoudt A, Victor J. Meniscal Allograft Transplantation Does Not Prevent or Delay Progression of Knee Osteoarthritis. PLoS One 2016;11:e0156183.

Virén T, Saarakkala S, Jurvelin JS, Pulkkinen HJ, Tiitu V, Valonen P, Kiviranta I, Lammi MJ, Töyräs J. Quantitative evaluation of spontaneously and surgically repaired rabbit articular cartilage using intra-articular ultrasound method in situ. Ultrasound Med Biol 2010;36:833-9.

Virén T, Saarakkala S, Kaleva E, Nieminen HJ, Jurvelin JS, Töyräs J. Minimally invasive ultrasound method for intra-articular diagnostics of cartilage degeneration. Ultrasound Med Biol 2009;35:1546-54.

Viren T, Saarakkala S, Tiitu V, Puhakka J, Kiviranta I, Jurvelin JS, Töyräs J. Ultrasound evaluation of mechanical injury of bovine knee articular cartilage under arthroscopic control. IEEE Trans Ultrason Ferroelectr Freq Control 2011;58:148-155.

Wareluk P, Szopinski KT. Value of modern sonography in the assessment of meniscal lesions. Eur J Radiol Elsevier Ireland Ltd, 2012;81:2366-2369.

Yasura K, Mizuno Y, Nakagawa Y, Mori K, Takenaka M, Ohashi T, Yamada K, Kobayashi M, Ando K, Kuroki H, Suzuki T, Ikeuchi K, Tsutsumi S, Nakamura T. Estimation of the Mechanical Property of MeniscusUsing Ultrasound: Examinations of Native Meniscusand Effects of Enzymatic 
Digestion. J Orthop Res 2007;25:884-893. 


\section{Tables}

Table 1. Spearman correlation coefficients between the quantitative ultrasound parameters (determined with $9 \mathrm{MHz}$ and $40 \mathrm{MHz}$ ultrasound) and composition or mechanical properties of the 5 meniscus.

\begin{tabular}{lccccccc}
\hline $\mathbf{9 M H z}$ & $R$ & $I R C$ & $U R I$ & $A I B$ & $A l B s$ & $A I B m$ & $A I B d$ \\
\hline$E_{\text {eq }}$ & 0.15 & 0.16 & -0.12 & -0.04 & 0.02 & 0.08 & -0.12 \\
$E_{\text {inst }}$ & 0.09 & 0.10 & 0.08 & -0.11 & -0.07 & -0.17 & -0.15 \\
$\mathrm{H}_{2} \mathrm{O}$ & -0.03 & 0.04 & -0.11 & 0.12 & 0.06 & 0.11 & 0.10 \\
$\mathrm{OD}$ & -0.02 & -0.01 & 0.01 & -0.11 & -0.10 & -0.12 & -0.11 \\
$U A$ & $0.53^{* *}$ & $0.52^{* *}$ & 0.01 & 0.07 & 0.04 & -0.13 & 0.05 \\
$\mathrm{HyPro}$ & $-0.36^{* *}$ & $-0.36^{* *}$ & 0.03 & 0.21 & 0.11 & $0.24^{*}$ & $0.23^{*}$ \\
\hline $\mathbf{4 0} \mathrm{MHz}$ & & & & & & & \\
$E_{\text {eq }}$ & -0.11 & -0.06 & -0.17 & $-0.37^{* *}$ & $0.26^{*}$ & $-0.25^{*}$ & $0.22^{*}$ \\
$E_{\text {inst }}$ & 0.03 & 0.05 & 0.02 & $-0.30^{* *}$ & -0.21 & -0.16 & -0.12 \\
$\mathrm{H}_{2} \mathrm{O}$ & -0.04 & -0.08 & -0.03 & -0.09 & 0.12 & 0.01 & 0.01 \\
$O D$ & $0.29 *$ & 0.27 & -0.17 & $-0.45^{* *}$ & $0.42^{* *}$ & $-0.27^{*}$ & -0.13 \\
$U A$ & 0.11 & 0.13 & -0.16 & $-0.33^{*}$ & -0.20 & $-0.29 *$ & $-0.39 * *$ \\
$H y P r o$ & $-0.24^{*}$ & $-0.16^{*}$ & $0.40^{* *}$ & -0.01 & -0.03 & 0.08 & 0.21 \\
\hline \hline
\end{tabular}

${ }^{*} p<0.05 ;{ }^{* *} p<0.01 ; R=$ Reflection coefficient; IRC = Integrated reflection coefficient; $U R I=$ Ultrasound roughness index; $A I B, A I B s, A I B m$ and $A I B d=$ Apparent integrated backscattering parameters; $E_{e q}=$ Equilibrium modulus; $E_{\text {inst }}=$ Instantaneous modulus; $\mathrm{H}_{2} \mathrm{O}=$ water content; $O D=$ optical density; UA = Uronic acid; HyPro = Hydroxyproline 
Table 2. Reproducibility ( $C V$ and $S C V$ ) and Least Significant Change ( $L S C$ ) of the determined quantitative ultrasound parameters.

\begin{tabular}{|c|c|c|c|c|c|c|c|c|}
\hline & & $R$ & $I R C$ & $U R I$ & $A / B$ & AlBs & AIBm & $A / B d$ \\
\hline & $C V(\%)$ & 2.2 & 0.3 & 27.4 & 0.4 & 0.6 & 0.4 & 0.1 \\
\hline \multirow[t]{3}{*}{$40 \mathrm{MHz}$} & sCV (\%) & 2.0 & 0.8 & 18.1 & 1.5 & 1.5 & 1.4 & 0.5 \\
\hline & LSC (\%) & 5.5 & 2.4 & 51.1 & 4.1 & 4.3 & 4.1 & 1.5 \\
\hline & $C V(\%)$ & 0.9 & 0.1 & 13.5 & 0.8 & 0.9 & 1.1 & 0.8 \\
\hline \multirow[t]{2}{*}{$9 \mathrm{MHz}$} & $s C V(\%)$ & 0.6 & 0.2 & 8.8 & 1.2 & 1.9 & 1.9 & 1.2 \\
\hline & LSC (\%) & 1.8 & 0.5 & 24.8 & 3.4 & 5.3 & 5.2 & 3.5 \\
\hline
\end{tabular}

$5 A I B, A I B s, A I B m$ and $A I B d=$ Apparent integrated backscattering parameters 
Table 3. $p$-values of linear relation of quantitative ultrasound parameters (dependent variables) and composition and mechanical properties of meniscus (fixed variables) using mixed model analysis. Statistically significant values are bolded.

$9 \mathrm{MHz}$

\begin{tabular}{lccccccc}
\hline Fixed/dependent & $R$ & $I R C$ & $U R I$ & $A l B$ & $A l B s$ & $A I B m$ & $A I B d$ \\
\hline$E_{\text {eq }}$ & 0.63 & 0.72 & 0.36 & 0.52 & 0.92 & 0.95 & 0.73 \\
$E_{\text {inst }}$ & 0.26 & 0.40 & 0.61 & 0.53 & 0.57 & 0.14 & 0.18 \\
$\mathrm{H}_{2} \mathrm{O}$ & $\mathbf{0 . 0 0}$ & $\mathbf{0 . 0 0}$ & 0.16 & 0.55 & 0.25 & 0.84 & 0.98 \\
OD & 0.19 & 0.22 & 0.72 & 0.41 & 0.49 & 0.12 & 0.52 \\
$U A$ & $\mathbf{0 . 0 1}$ & $\mathbf{0 . 0 1}$ & 0.68 & 0.65 & 0.60 & 0.46 & 0.08 \\
$\mathrm{HyPro}$ & 0.18 & $\mathbf{0 . 0 3}$ & $\mathbf{0 . 0 2}$ & 0.16 & 0.33 & 0.27 & $\mathbf{0 . 0 2}$ \\
\hline $\mathbf{4 0 ~} \mathbf{M H z}$ & & & & & & & \\
$E_{\text {eq }}$ & 0.95 & 0.83 & $\mathbf{0 . 0 0}$ & 0.24 & 0.13 & 0.75 & 0.31 \\
$E_{\text {inst }}$ & 0.30 & 0.17 & $\mathbf{0 . 0 0}$ & 0.21 & 0.32 & 0.57 & 0.09 \\
$\mathrm{H}_{2} \mathrm{O}$ & 0.53 & 0.93 & 0.90 & 0.93 & 0.67 & 0.43 & 0.12 \\
OD & 0.10 & 0.14 & 0.36 & $\mathbf{0 . 0 5}$ & 0.08 & 0.25 & 0.28 \\
UA & 0.21 & 0.10 & 0.15 & 0.08 & 0.16 & 0.22 & 0.13 \\
HyPro & 0.82 & 0.79 & $\mathbf{0 . 0 0}$ & 0.17 & 0.20 & 0.92 & 0.51 \\
\hline \hline
\end{tabular}

$R=$ Reflection coefficient; $I R C$ = Integrated reflection coefficient; $U R I$ = Ultrasound roughness index;

$5 A I B, A l B s, A I B m$ and $A l B d=$ Apparent integrated backscattering parameters; $E_{\text {eq }}=$ Equilibrium modulus; $E_{\text {inst }}=$ Instantaneous modulus; $\mathrm{H}_{2} \mathrm{O}=$ water content; $O D=$ optical density; $U A=$ Uronic acid; HyPro = Hydroxyproline 
Table 4. Mean and standard deviation (SD) of the measured prediction variables and corresponding statistics of variables predicted from ultrasound backscattering envelope and power spectrum (9 and $40 \mathrm{MHz}$ ultrasound transducers) data with PLS regression analysis.

\begin{tabular}{|c|c|c|c|c|c|c|c|c|}
\hline \multirow{2}{*}{$\begin{array}{l}\mathbf{9 M H z} \\
\text { Response variable }\end{array}$} & \multirow[b]{2}{*}{ mean } & \multirow[b]{2}{*}{ SD } & \multicolumn{3}{|c|}{ Hilbert envelope } & \multicolumn{3}{|c|}{ Power spectrum } \\
\hline & & & $n$ & $R^{2}$ & RMSECV & $n$ & $R^{2}$ & RMSECV \\
\hline$E_{e q}(\mathrm{MPa})$ & 0.17 & 0.11 & 4 & $0.06^{*}$ & 0.11 & 5 & $0.20 * *$ & 0.10 \\
\hline$E_{\text {inst }}(\mathrm{MPa})$ & 0.64 & 0.47 & 6 & $0.15^{* *}$ & 0.44 & 6 & $0.20 * *$ & 0.44 \\
\hline $\mathrm{H}_{2} \mathrm{O}(\%)$ & 75.04 & 3.07 & 5 & $0.11^{* *}$ & 2.97 & 6 & $0.18 * *$ & 2.82 \\
\hline$O D(A U)$ & 0.37 & 0.24 & 4 & $0.08 * *$ & 0.23 & 5 & $0.10 * *$ & 0.23 \\
\hline$U A(\mu \mathrm{g} / \mathrm{mg})$ & 1.28 & 0.61 & 4 & $0.13 * *$ & 0.57 & 6 & $0.24^{* *}$ & 0.53 \\
\hline HyPro ( $\mu \mathrm{g} / \mathrm{mg})$ & 14.11 & 4.51 & 4 & $0.15^{* *}$ & 4.25 & 6 & $0.26 * *$ & 3.96 \\
\hline \multicolumn{9}{|l|}{$40 \mathrm{MHz}$} \\
\hline$E_{e q}(\mathrm{MPa})$ & 0.17 & 0.11 & 6 & $0.49 * *$ & 0.08 & 6 & $0.48 * *$ & 0.08 \\
\hline$E_{\text {inst }}(\mathrm{MPa})$ & 0.64 & 0.47 & 6 & $0.56 * *$ & 0.31 & 6 & $0.40 * *$ & 0.37 \\
\hline $\mathrm{H}_{2} \mathrm{O}(\%)$ & 75.04 & 3.07 & 4 & $0.38 * *$ & 2.41 & 5 & $0.30 * *$ & 2.65 \\
\hline$O D(A U)$ & 0.37 & 0.24 & 6 & $0.52 * *$ & 0.17 & 3 & $0.50 * *$ & 0.17 \\
\hline$U A(\mu \mathrm{g} / \mathrm{mg})$ & 1.28 & 0.61 & 6 & $0.63 * *$ & 0.38 & 6 & $0.40 * *$ & 0.49 \\
\hline HyPro ( $\mu \mathrm{g} / \mathrm{mg})$ & 14.11 & 4.51 & 5 & $0.69 * *$ & 2.50 & 6 & $0.51^{* *}$ & 3.17 \\
\hline
\end{tabular}

$5 n=$ Number of PLS components in PLS regression model, $R^{2}=$ Coefficient of determination, $R M S E C V$

$=$ Root mean square error of cross-validation, $E_{e q}=$ Equilibrium modulus, $E_{\text {inst }}=$ Instantaneous modulus, $\mathrm{H}_{2} \mathrm{O}=$ Water content, $O D=$ Optical density, $U A=$ Uronic acid, HyPro $=$ Hydroxyproline, $\mathrm{AU}$ $=$ Arbitrary unit, $* * p<0.01, * p<0.05$ 
Figures
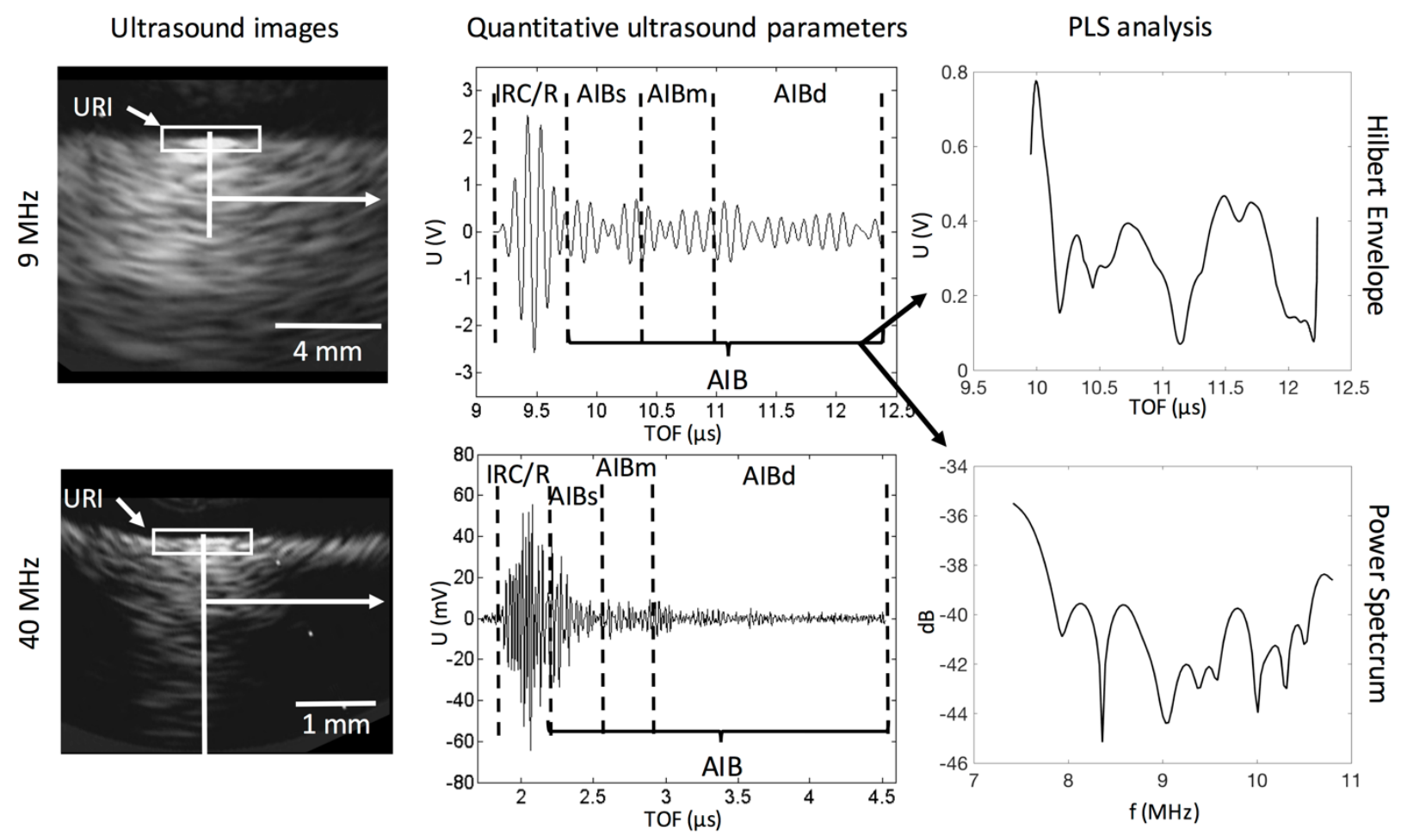

Figure 1. For the calculations of quantitative ultrasound parameters one scanline perpendicular to meniscus surface was selected in to analysis (white line). Subsequently the ultrasound RF-signal was windowed using a hamming window $(R$ and $I R C)$ or a rectangular window $(A I B, A I B s, A I B m$ and $A l B d)$. For the PLS analysis Hilbert envelope and power spectrum normalized with reference spectrum were determined from windowed ultrasound backscattering signal measured perpendicular to meniscus surface ( $A / B$ window). Subsequently, envelope and spectrum values were used as variables in the multivariate PLS analysis. Ultrasound roughness index (URI) was calculated from meniscus surface profile determined based on 21 points adjacent to perpendicular scanline (white box). 
$9 \mathrm{MHz}$
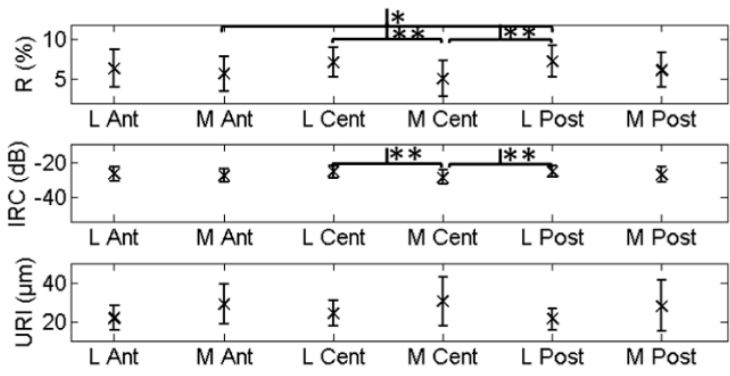

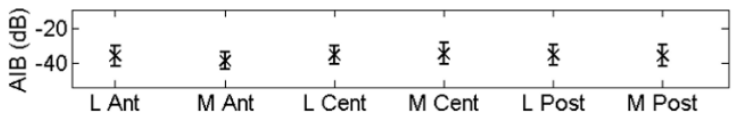

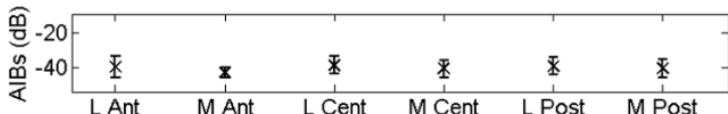

产-20

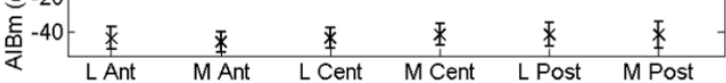

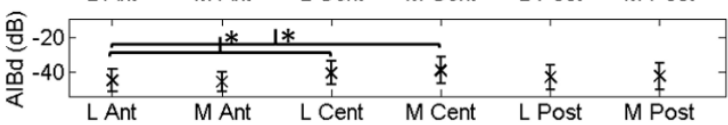

$40 \mathrm{MHz}$
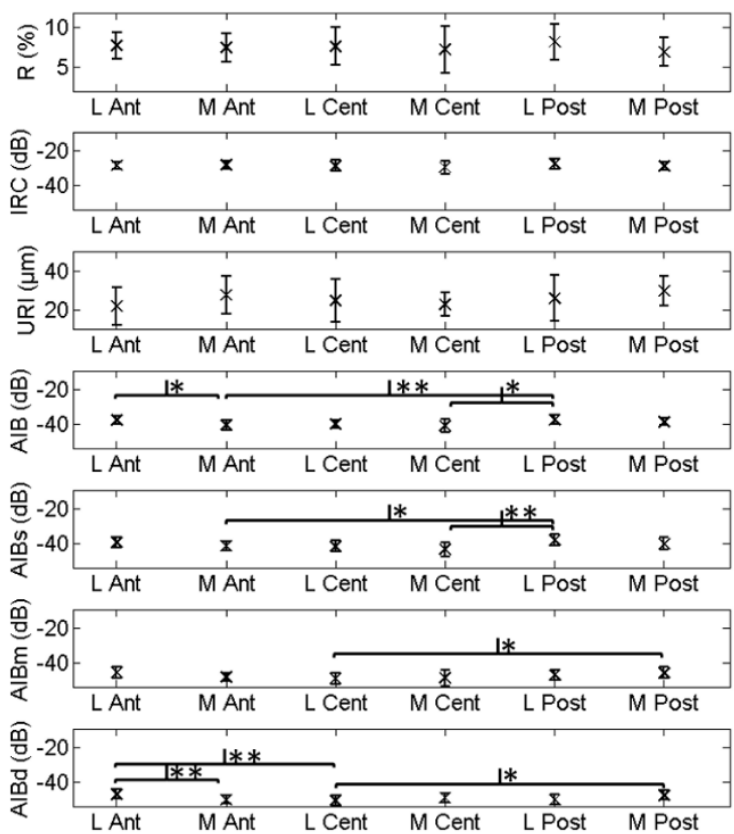

Figure 2. Quantitative ultrasound parameters determined with $9 \mathrm{MHz}$ and $40 \mathrm{MHz}$ ultrasound from anterior horn ( $L$ ant and $M$ ant), central meniscus ( $L$ cent and $M$ cent), and posterior horn ( $L$ post and $\mathrm{M}$ post) of medial and lateral meniscus. ${ }^{*} p<0.05,{ }^{* *} p<0.01$, Freedman test 